\title{
Bioinoculantes y concentración de la solución nutritiva sobre la producción y calidad de tomate
}

\author{
Bioinoculants and concentration of the nutritive solution on the production and quality of tomato
}

\begin{abstract}
Bernardo Espinosa-Palomeque ${ }^{1}$, Pedro Cano-Ríos¹, Lilia Salas-Pérez², José Luis García-Hernández ${ }^{3}$, Pablo PreciadoRangel ${ }^{1}$, Jorge Sáenz-Mata ${ }^{3}$ y José Luis Reyes-Carrillo ${ }^{1 *}$

Universidad Autónoma Agraria Antonio Narro Unidad Laguna. Periférico Raúl López Sánchez km 1.5 y Carretera a Santa Fe S/N. 27059 Torreón, Coahuila, México.

2 Universidad Politécnica de Gómez Palacio. Carretera El Vergel - La Torreña, km 0.820.35120 Gómez Palacio, Durango, México. Universidad Juárez del Estado de Durango, Av. Universidad s/n Fracc. Filadelfia, 35010, Gómez Palacio, Durango, México.
\end{abstract}

\section{RESUMEN}

El objetivo del presente estudio fue evaluar dos rizobacterias promotoras del crecimiento vegetal [RPCV (Bacillus paralicheniformis y Pseudomonas lini)] inoculadas individual y co-inoculadas con dos concentraciones de solución nutritiva (SN) 75 y $100 \%$ sobre la productividad y calidad de tomate en invernadero. Se compararon ocho tratamientos: $\mathrm{T} 1=B$. paralicheniformis + SN $75 \%, \mathrm{~T} 2=P$. lini + SN $75 \%, \mathrm{~T} 3=$ co-inoculante + SN $75 \%$, T4 = sin RPCV + SN $75 \%, \mathrm{~T} 5=$ B. paralicheniformis + SN $100 \%$, T6= P. lini + SN $100 \%$, T7 = co-inoculante + SN $100 \%$ y T8= sin RPCV + SN $100 \%$. El experimento se estableció en un diseño bloques al azar con 12 repeticiones. Los resultados indicaron que con el tratamiento $\mathrm{T} 1$ se obtuvieron los mayores valores para el rendimiento ( $2.09 \mathrm{~kg} / \mathrm{planta})$. El tratamiento T3 incrementó la capacidad antioxidante (46.19 $\mu \mathrm{M}$ ETrolox/100 g peso fresco) de los frutos con relación a los tratamientos sin RPCV (T4 y T8). El uso de RPCV puede ser una alternativa sostenible que permite disminuir la fertilización para mejorar la calidad sin disminuir el rendimiento de fruto, además de mitigar los efectos adversos de los fertilizantes inorgánicos en los sistemas de producción del cultivo de tomate.

Palabras claves: agricultura protegida, calidad nutracéutica, rendimiento, RPCV

\section{ABSTRACT}

The objective of the present study was to evaluate two plant growth promoting rhizobacteria [PGPR (Bacillus paralicheniformis and Pseudomonas lini)] inoculated individually and co-inoculated with two concentrations of nutrient solution (SN), 75 and $100 \%$, on the productivity and quality of tomato in greenhouse. Eight treatments were compared:

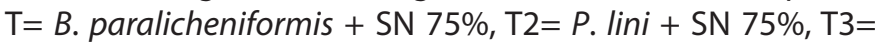
co-inoculant + SN 75\%, T4= without PGPR + SN 75\%, T5=B. paralicheniformis + SN 100\%, T6= P. lini + SN 100\%, T7 = co-inoculant + SN $100 \%$ and T8 $=$ without PGPR + SN 100\%. The experiment was established in a randomized blocks design with 12 repetitions. The results indicated that with the T1 treatment the highest yield values were obtained $(2.09 \mathrm{~kg} /$ plant). The T3 treatment increased the fruits antioxidant capacity (46.19 $\mathrm{MM}$ ETrolox/100 $\mathrm{g}$ fresh weight) in relation to the

\section{Volumen XXI, Número 3}

treatments without PGPR (T4 y T8). The use of PGPR can be a sustainable alternative that allows reducing fertilization to improve quality without diminishing the fruit yield, besides mitigating the adverse effects of the inorganic fertilizers in the production systems of the tomato crop.

Keywords: protected agriculture, nutraceutical quality, PGPR, yield

\section{INTRODUCCIÓN}

El tomate (Solanum lycopersicum L.) es uno de los cultivos hortícolas más cultivado en las regiones tropicales y subtropicales del mundo. China es el principal productor con 54.8 millones de ton producidas. México ocupa el décimo lugar con una producción de 4 millones de ton (FAOSTAT, 2016). El fruto de tomate es una buena fuente dietética de vitaminas ( $A, C, E)$, ácido fólico, ascorbato, polifenoles, carotenoides y otros nutrientes esenciales. Estos compuestos presentan actividad antioxidante que ayudan a reducir los riesgos de cáncer y enfermedades cardiovasculares (Al-Harbi et al., 2017).

La superficie dedicada para la producción del cultivo de tomate se encuentra en continua expansión principalmente en ambientes protegidos, al mismo tiempo el uso de agroquímicos, particularmente, los fertilizantes inorgánicos los cuales sin un adecuado suministro pueden causar problemas en la salud humana y al ambiente (Salas-Perez et al., 2016). Una alternativa sostenible para disminuir los impactos negativos de los fertilizantes inorgánicos y aumentar la calidad de los frutos es la aplicación de bioinoculantes a base de rizobacterias promotoras del crecimiento vegetal (RPCV) (Kloepper y Schroth, 1978) los cuales son económicos, no contamina al ambiente, contribuyen a la conservación de la fertilidad y biodiversidad del suelo, además, pueden suministrarse con dosis reducidas de fertilizantes inorgánicos (Chiquito-Contreras et al., 2017).

Los géneros más importantes de RPCV incluyen Acinetobacter, Aeromonas, Alcaligenes, Arthrobacter, Azospirillum, Bacillus, Burkholderia, Enterobacter, Erwinia, Flavobacterim, Pseudomonas, Rhizobium, Serratia, entre otros (Glick, 2012). Estos grupos de microorganismos colonizan las raíces de las plantas y mejoran el crecimiento de las plantas ya sea por

*Autor para correspondencia: José Luis Reyes-Carrillo Correo electrónico: jlreyes54@gmail.com

Recibido: 7 de noviembre de 2018 Aceptado: 14 de junio de 2019 
mecanismos directos o indirectos (Shameer y Prasad, 2018). Los mecanismos directos son la producción de fitohormonas, fijación biológica de nitrógeno, disminución de la síntesis del etileno, la solubilización de nutrimentos como $\mathrm{P}, \mathrm{K}$ y Fe (Olanrewaju et al., 2017). Los mecanismos indirectos es la inhibición de uno o más fitopatógenos mediante la síntesis de antibióticos y sideróforos, la resistencia sistémica inducida y resistencia sistémica adquirida (Singh et al., 2017).

El uso de RPCV en combinación con fertilizantes inorgánicos mejoran el crecimiento de las plantas debido a un incremento en la absorción de nutrimentos (Adesemoye et al., 2009). Dicho aumento se relaciona a que los fertilizantes inorgánicos incrementan las poblaciones de RPCV que producen principalmente ácido indol-3-acético y sideróforos en el medio de crecimiento (Yuan et al., 2010; ChiquitoContreras et al., 2017). Aunado a lo anterior, la aplicación de los géneros rizosféricos Bacillus (B. amyloliquefaciens IN937a y B. pumilus T4) y Pseudomonas (Pseudomonas sp. 19Fv1T y $P$. fluorescens) con dosis reducidas del 50 y $30 \%$ de la fertilización inorgánica han aumentado el crecimiento, rendimiento, la calidad organoléptica y nutracéutica de frutos de tomate desarrollados en condiciones de invernadero y campo, respectivamente (Toor y Savage, 2005; Bona et al., 2018). Con base en lo anteriormente señalado, el objetivo del presente estudio fue evaluar dos RPCV (B. paralicheniformis y $P$. lini) inoculadas individual y co-inoculadas con dos concentraciones de solución nutritiva 75 y $100 \%$ sobre la productividad y calidad de tomate en condiciones de invernadero.

\section{MATERIALES Y MÉTODOS Sitio de estudio}

Esta investigación se realizó en el ciclo agrícola primavera-verano 2017, en un invernadero con enfriamiento automático, en la Universidad Autónoma Agraria Antonio Narro Unidad Laguna, Torreón, Coahuila, México, en las coordenadas $25^{\circ} 33^{\prime} 26^{\prime \prime} \mathrm{N}$ y $103^{\circ} 22^{\prime} 31^{\prime \prime} \mathrm{O}$; a una altitud de 1230 $\mathrm{m}$. Durante los 120 días del ciclo del cultivo, la temperatura minina y máxima en el interior del invernadero fluctuó entre 17.7 y $31.6^{\circ} \mathrm{C}$, respectivamente, mientras la humedad relativa mínima y máxima osciló entre 30 y $70 \%$.

\section{Propagación de RPCV}

Se utilizaron dos cepas B. paralicheniformis y P. lini perteneciente a la colección microbiana del Laboratorio de Ecología Microbiana de la Facultad de Ciencias Biológicas de la Universidad Juárez del Estado de Durango. Las cuales se han caracterizado como promotoras de crecimiento, ya sea por la producción de ácido indol acético (AIA), de sideróforos, actividad de la enzima del ácido 1-aminociclopropano1-carboxílico (ACC) desaminasa o solubilizadoras de fosfato (Palacio-Rodríguez et al., 2017). Las cepas se propagaron individualmente en medio Luria Bertani en una incubadora con agitación de $200 \mathrm{rpm}$ (Precision Scientific $815^{\circ}$ ) durante $24 \mathrm{~h}$ a $30{ }^{\circ} \mathrm{C}$, posteriormente la concentración bacteriana se ajustó a $1 \times 10^{8} \mathrm{UFC} / \mathrm{mL}$ con buffer fosfato salino al $0.5 \mathrm{x}$.

\section{Material genético e inoculación de las plantas}

Se sembraron semillas de tomate cv. Moctezuma (Harris Moran ${ }^{\circ}$ ) en charolas de poliestireno de 200 cavidades, las cuales contenían Peat moss (Premier, México) como sustrato. Para mantener la humedad se cubrieron con plástico negro durante $72 \mathrm{~h}$ y se aplicaron riegos cada $24 \mathrm{~h}$. Para la inoculación de las raíces de las plántulas de 12 días de emergidas se empleó el método de inmersión durante 5 min en la suspensión bacteriana. Los tratamientos testigos (T4 y T8) solo se trataron con agua destilada (González et al., 2018). La inoculación para $B$. paralicheniformis y $P$. lini se realizó de forma individual, además, a las plantas de los tratamientos denominados co-inoculante (T3 y T7) se inocularon con las dos RPCV.

\section{Trasplante y manejo del cultivo}

El trasplante se realizó 35 días después de la siembra colocando una planta por maceta. Como maceta se utilizaron bolsas de polietileno negro calibre 500, con una capacidad de $18 \mathrm{~L}$. Las bolsas se llenaron con una mezcla a base de arena de río y perlita [Multiperl' ${ }^{\circ}$, México $\left.(80: 20, \mathrm{v}: \mathrm{v})\right]$. Las bolsas se colocaron en doble hilera, con un arreglo topológico en tresbolillo a una distancia de $0.30 \mathrm{~m}$ entre planta y $1.60 \mathrm{~m}$ entre hilera con una densidad de 4.2 plantas $/ \mathrm{m}^{2}$. La arena de río se desinfectó previamente con una solución de $5 \%$ de hipoclorito de sodio y se dejó secar al ambiente por tres días. A los cuatro días después del trasplante (ddt) se inició el riego aplicando la SN al 75 y $100 \%$. Las SNs fueron preparadas a partir de nitrato de calcio $\left.\left[\mathrm{Ca}(\mathrm{NO}]_{3}\right)_{2} \cdot 4 \mathrm{H}_{2} \mathrm{O}\right]$, nitrato de potasio $\left(\mathrm{KNO}_{3}\right)$, sulfato de magnesio $\left(\mathrm{MgSO}_{4} \cdot 7 \mathrm{H}_{2} \mathrm{O}\right)$, sulfato de potasio $\left(\mathrm{K}_{2} \mathrm{SO}_{4}\right)$, más micronutrimentos (Maxiquel $\left.{ }^{\circ}\right)$. El pH de las soluciones se ajustó a 5.5 con ácido fosfórico $\left(\mathrm{H}_{3} \mathrm{PO}_{4}\right)$. La necesidad hídrica del cultivo se cubrió aplicando riegos de manera manual considerando tres etapas de desarrollo del cultivo a) antes de la floración, b) floración y c) producción, en las cuales se aplicaron un volumen de $0.5,1$ y 2 L/día, respectivamente. El agua de riego tuvo una conductividad eléctrica de $1.18 \mathrm{dS} \mathrm{m}^{-1}$, RAS de $3.2, \mathrm{pH}$ de 7.8 , por lo que se clasificó como agua de baja salinidad y bajo contenido de sodio (Ayers y Westcot, 1994).

La cosecha de los frutos se realizó del primer al octavo racimo, cuando éstos presentaron un color rosa entre 30 y 60 $\%$ de acuerdo a la clasificación de color de USDA (1991).

\section{Diseño experimental}

Se utilizó un diseño experimental de bloques al azar con ocho tratamientos con 12 repeticiones. Los tratamientos fueron la combinación de RPCV y niveles de la concentración de la solución nutritiva Steiner (SN) (Steiner, 1961): $\mathrm{T} 1=$ B. paralicheniformis $+\mathrm{SN} 75 \%, \mathrm{~T} 2=P$. lini $+\mathrm{SN} 75 \%, \mathrm{~T} 3=$ co-inoculante + SN $75 \%, \mathrm{~T} 4=\sin \mathrm{RPCV}+\mathrm{SN} 75 \%, \mathrm{~T} 5=B$. paralicheniformis + SN $100 \%, \mathrm{~T} 6=P$. lini + SN $100 \%, \mathrm{~T} 7=\mathrm{CO}-$ inoculante + SN $100 \%$ y T8 $=\sin$ RPCV + SN $100 \%$. La unidad experimental fue una planta de un solo tallo por maceta. 
Altura de planta, diámetro de tallo y número de hojas

La altura de planta se midió desde la base del tallo hasta la yema apical con un flexómetro (Truper', México), el diámetro de tallo se determinó a un $1 \mathrm{~cm}$ de la base del tallo con un vernier digital (Truper, México) y se contó el número de hojas por planta, estas variables se evaluaron al final del ciclo del cultivo.

\section{Rendimiento y calidad biofísica}

Para determinar el efecto que inducen los tratamientos sobre la calidad biofísica de los frutos, se evaluaron las variables: tamaño del fruto (diámetro polar y diámetro ecuatorial) y el espesor de pericarpio utilizando un vernier, el contenido de sólidos solubles (determinado en ${ }^{\circ} B r i x$, con un refractómetro Master-T ATAGO', Tokio, Japón), la firmeza con un penetrómetro con embolo de $3 \mathrm{~mm}$ (FHT200, Extech Instruments ${ }^{\circ}$, USA), el peso promedio del fruto (utilizando una báscula Ohaus $3729^{\circ}$, México); todas estas variables se determinaron en 15 frutos por planta, correspondiente a cada repetición de cada tratamiento. El rendimiento se obtuvo por planta, del primero al octavo racimo (Rodríguez et al., 2007).

Para la determinación de fenoles totales, flavonoides y capacidad antioxidante, se mezclaron $2 \mathrm{~g}$ de muestra de fruto fresco con $10 \mathrm{~mL}$ de etanol en tubos de plástico con tapón de rosca. Se usó un agitador tipo "Stuart" para mantener la mezcla en agitación durante $24 \mathrm{~h}$ a $30^{\circ} \mathrm{C}$. Posteriormente, los tubos se centrifugaron a $3000 \mathrm{rpm}$ durante $5 \mathrm{~min}$ y los sobrenadantes (extracto etanólico) fueron utilizados para su análisis respectivo.

\section{Fenoles totales}

El contenido de fenoles totales se determinó usando una modificación del método descrito por Toor y Savage (2005). Doscientos $\mu \mathrm{L}$ de extracto etanólico se mezclaron con $3 \mathrm{~mL}$ de agua destilada, posteriormente se le añadieron 250 $\mu \mathrm{L}$ del reactivo Folin-Ciocalteu (Sigma-Aldrich', St Louis MO, USA), se agitó con un vortex. Después de $3 \mathrm{~min}$ de reposo se añadieron $750 \mu \mathrm{L}$ de carbonato de sodio $(20 \% \mathrm{p} / \mathrm{v})$ y se agitó durante $10 \mathrm{~s}$. La solución se dejó reposar a temperatura ambiente durante $30 \mathrm{~min}$ en oscuridad. La absorbancia de la solución se leyó a $750 \mathrm{~nm}$ en un espectrofotómetro Genesys 10UV (Madison', Wisconsin USA). Se calculó el contenido fenólico mediante una curva de calibración utilizando ácido gálico (Sigma-Aldrich', St Louis MO, USA) como estándar. Los resultados se registraron en $\mathrm{mg}$ de equivalente de ácido gálico por $100 \mathrm{~g}$ peso fresco (mg EAG/ $100 \mathrm{~g}$ peso fresco).

\section{Flavonoides totales}

El contenido de flavonoides totales se determinó usando el método colorimétrico propuesto por Zhishen et al. (1999). Para ello se mezclaron $500 \mu \mathrm{L}$ de muestra del extracto etanólico con $1.25 \mathrm{~mL}$ de agua destilada, seguido se adicionaron $75 \mu \mathrm{L}$ de $\mathrm{NaNO}_{2}$ al $5 \%$. Después de 5 min de reposo se añadió $150 \mu \mathrm{L}$ de $\mathrm{AlCl}_{3}$ al $10 \%$ y se dejó reposar durante 6 min. Posteriormente, se añadieron $500 \mu \mathrm{L}$ de $\mathrm{NaOH} 1 \mathrm{M}$ y 275 $\mu \mathrm{L}$ de agua destilada. Todos los componentes se mezclaron con un vortex. La absorbancia se midió inmediatamente a $510 \mathrm{~nm}$ usando el espectrofotómetro (Genesys 10UV). Los resultados obtenidos se expresaron en $\mathrm{mg}$ de equivalentes de quercetina por $100 \mathrm{~g}$ peso fresco (mg EQ/100 $\mathrm{g}$ peso fresco).

\section{Capacidad antioxidante}

La capacidad antioxidante se determinó basándose en el método descrito por Brand-Williams et al. (1995) con algunas modificaciones. Se preparó una solución de radical libre 1.1-difenil-2-picrilhidrazil $\left(\mathrm{DPPH}^{+}\right)$(Sigma-Aldrich', St Louis MO, USA) en etanol ( $5 \mathrm{mg} / 100 \mathrm{~mL}$ de etanol grado analítico), en un matraz cubierto con papel aluminio. La mezcla se agitó vigorosamente y el matraz se mantuvo cubierto para evitar la degradación rápida. La prueba de capacidad antioxidante se realizó mezclando $100 \mu \mathrm{L}$ de muestra del extracto etanólico y $1.9 \mathrm{~mL}$ de $\mathrm{DPPH}^{+}$. Las lecturas se realizaron a $517 \mathrm{~nm}$ después de 90 min de incubación. La capacidad antioxidante total se calculó utilizando una curva estándar con el antioxidante de referencia Trolox y los resultados se expresaron en $\mu \mathrm{M}$ equivalentes Trolox por $100 \mathrm{~g}$ de peso fresco $(\mu \mathrm{M}$ ETrolox/100 $\mathrm{g}$ peso fresco). Los análisis se realizaron por triplicado.

\section{Análisis estadístico}

Se realizó un análisis de varianza para determinar las diferencias entre los tratamientos y la prueba múltiple de Tukey $(P \leq 0.05)$ para la comparación de medias. Los análisis se realizaron con el software estadístico Statistical Analysis System 9.0 (SAS, 2004).

\section{RESULTADOS Y DISCUSIÓN \\ Altura de planta, diámetro de tallo y número de hojas}

Los resultados mostraron que la inoculación de RPCV y concentraciones de solución nutritiva influyeron en altura de planta, diámetro de tallo y número de hojas $(P<0.01)$ (Tabla 1). Los mayores valores en altura de planta se encontraron con el tratamiento T6 con $281.41 \mathrm{~cm}$, superando en 10.86 y $3.40 \%$ a los tratamientos T4 y T8 (testigos), respectivamente. Le siguieron los tratamientos T3, T2 y T7 con valores de 278, 276.92 y $276 \mathrm{~cm}$, respectivamente. En cuanto al número de hojas, en el tratamiento T6 se obtuvo diferencia significativa y un aumento del $10.34 \%$ en comparación al T4. Aunque el número de hojas registradas en T6 fue numéricamente mayor que las mostradas al resto de los tratamientos, no hubo diferencia significativa entre ellos.

En conjunto, los resultados del presente estudio fortalecen la hipótesis de que los biofertilizantes a base de RPCV promueven el crecimiento de las plantas al aumentar las cantidades de nutrimentos absorbidos por las raíces y una acumulación en los tejidos vegetales. Ello se logra a través de una mayor disponibilidad de nutrimentos en la rizosfera (Abraham-Juárez et al., 2018). Así también al mejorar la membrana plasmática involucrada en el proceso de nutrición a nivel del sistema radical al alterar la selectividad de $\mathrm{Na}^{+}, \mathrm{K}^{+} \mathrm{y}$ $\mathrm{Ca}^{2+}$ y mantienen una mayor proporción de $\mathrm{K}^{+} / \mathrm{Na}^{+}$en plantas bajo estrés salino (Vacheron et al., 2013; Bharti et al., 2016). Plantas de tomate inoculadas con $P$. putida y fertilizadas con 
Tabla 1. Valores promedio obtenidos en altura de planta, diámetro de tallo y número de hojas del tomate cultivado con diferentes niveles de solución nutritiva y rizobacterias promotoras del crecimiento vegetal.

Table 1. Average values obtained for plant height, stem diameter and number of leaves of tomato grown with different levels of nutrient solution and plant growth promoting rhizobacteria.

\begin{tabular}{|c|c|c|c|}
\hline Tratamiento & $\begin{array}{c}\text { Altura de } \\
\text { planta }\end{array}$ & $\begin{array}{c}\text { Diámetro de } \\
\text { tallo }\end{array}$ & $\begin{array}{c}\text { Número de } \\
\text { hoja }\end{array}$ \\
\hline & $\mathrm{cm}$ & $\mathbf{m m}$ & \\
\hline $\begin{array}{l}\text { T1 = B. paralicheniformis } \\
+ \text { SN } 75 \%\end{array}$ & $264.33 \pm 6.43^{\mathrm{ab}}$ & $6.33 \pm 0.14^{\mathrm{ab}}$ & $31.67 \pm 0.84^{\mathrm{a}}$ \\
\hline $\mathrm{T} 2=$ P. lini + SN $75 \%$ & $276.92 \pm 4.12^{\mathrm{a}}$ & $6.33 \pm 0.14^{\mathrm{ab}}$ & $30.50 \pm 0.66^{\mathrm{ab}}$ \\
\hline $\begin{array}{l}\text { T3 = Co-inoculante + SN } \\
75 \%\end{array}$ & $278.00 \pm 5.57^{a}$ & $6.00 \pm 0.24^{b}$ & $30.33 \pm 0.76^{\mathrm{ab}}$ \\
\hline $\mathrm{T} 4=\mathrm{Sin} \mathrm{RPCV}+\mathrm{SN} 75 \%$ & $253.83 \pm 6.03^{b}$ & $6.42 \pm 0.14^{\mathrm{ab}}$ & $29.75 \pm 0.42^{\mathrm{ab}}$ \\
\hline $\begin{array}{l}\text { T5 }=\text { B. paralicheniformis } \\
+ \text { SN } 100 \%\end{array}$ & $272.58 \pm 4.12^{\mathrm{ab}}$ & $7.00 \pm 0.21^{\mathrm{a}}$ & $31.92 \pm 0.46^{a}$ \\
\hline $\mathrm{T} 6=P . \operatorname{lini}+\mathrm{SN} 100 \%$ & $281.42 \pm 2.51^{\mathrm{a}}$ & $6.17 \pm 0.11^{b}$ & $32.00 \pm 0.49^{a}$ \\
\hline $\begin{array}{l}\text { T7 = Co-inoculante }+ \text { SN } \\
100 \%\end{array}$ & $276.00 \pm 4.64^{\mathrm{a}}$ & $6.50 \pm 0.19^{\mathrm{ab}}$ & $31.75 \pm 0.60^{\mathrm{a}}$ \\
\hline $\begin{array}{l}\mathrm{T} 8=\mathrm{Sin} \mathrm{RPCV}+\mathrm{SN} \\
100 \%\end{array}$ & $272.17 \pm 3.75^{\mathrm{ab}}$ & $6.08 \pm 0.19^{b}$ & $29.00 \pm 0.52^{b}$ \\
\hline Significancia & ** & ** & ** \\
\hline DMSH & 20.53 & 0.78 & 2.63 \\
\hline
\end{tabular}

†Letras distintas en la misma columna indican diferencia significativa, según la prueba Tukey $(P \leq 0.05)$. Co-inoculante = inoculación Bacillus paralicheniformis + Pseudomonas lini, SN = solución nutritiva Steiner, ${ }^{* *}=$ altamente significativo $(P<0.01), \mathrm{DMSH}=$ diferencia mínima significativa honesta.

$\mathrm{N}: P: K(17: 17: 17)$ incrementaron su altura, número de hojas y el contenido de clorofila, lo cual podría aumentar la fotosíntesis total, así mejorar la producción de biomasa del cultivo, con respecto a plantas no inoculadas (Babu et al., 2015). Dichos incrementos son de importancia debido a que el nitrógeno es parte estructural de la molécula de clorofila, es el componente principal de proteínas esenciales para la formación de protoplasma, que conduce a la elongación celular, la división celular y en última instancia aumentar el crecimiento de las especies vegetales (Ramakrishnan y Selvakumar, 2012). Los resultados de este estudio y los reportados previamente por otros autores indican que una mayor altura de planta trae consigo incrementos en número de hojas, esto podría aumentar la fotosíntesis total, el peso del fruto y el rendimiento (Rodríguez et al., 2009).

En relación con el diámetro de tallo el tratamiento T5 presentó el mayor promedio con $7 \mathrm{~mm}$, superando significativamente un 16.7 y $15.13 \%$ a los tratamientos sin RPCV T4 y T8, respectivamente (Tabla 1). En los tratamientos con la dosis de fertilización reducida e inoculados con las RPCV (T1 al T3) se obtuvieron resultados similares a los de Xiao et al. (2015), quienes reportaron que cuando se redujo la dosis de fertilizantes inorgánicos y se usaron inoculantes a base de $B$. subtilis y $P$. fluorescens en el cultivo de tomate, la altura de planta y el diámetro de tallo fueron superiores o comparables a aquellos con fertilización completa sin inoculantes. Estos incrementos se atribuyen a que las RPCV aumentan la disponibilidad de nutrimentos en el medio radical, particularmente nitrógeno (fijación biológica de nitrógeno), fósforo (solubilización de fósforo) y hierro (producción de sideróforos), mejorando el desarrollo del sistema radical, aumentando la actividad enzimática de la planta o provocando una sinergia con microorganismos benéficos mejorando así su acción sobre la planta o inhibir sus patógenos (Tabassum et al., 2017).

\section{Rendimiento y calidad biofísica}

El número de fruto y rendimiento por planta mostraron diferencias estadísticas entre los tratamientos $(P<0.01)$. En contraste, el peso de fruto, firmeza y diámetro polar y diámetro ecuatorial no presentaron diferencias significativas $(P$ $>0.05$ ) (Tabla 2). El mayor número de frutos se registró en los

Tabla 2. Valores promedio obtenidos en calidad biofísica de tomate cultivado con diferentes concentraciones de solución nutritiva y rizobacterias promotoras del crecimiento vegetal. Table 2. Average values obtained in biophysical quality of tomato grown with different concentrations of nutrient solution and plant growth promoting rhizobacteria.

\begin{tabular}{lcccccc}
\hline Tratamiento & PF & DP & DE & F & NF & RP \\
\cline { 2 - 7 } & $\mathbf{g}$ & \multicolumn{2}{c}{$\mathbf{c m}$} & $\mathbf{N}$ & & kg planta $^{-1}$ \\
\hline T1 & $61.27 \pm 2.25^{\dagger}$ & $5.61 \pm 0.09$ & $4.34 \pm 0.07$ & $11.72 \pm 0.15$ & $33.33 \pm 1.23^{\text {a }}$ & $2.04 \pm 0.09^{\mathrm{a}}$ \\
T2 & $59.81 \pm 1.85$ & $5.62 \pm 0.09$ & $4.30 \pm 0.04$ & $11.83 \pm 0.15$ & $31.00 \pm 0.97^{\mathrm{ab}}$ & $1.85 \pm 0.06^{\mathrm{a}}$ \\
T3 & $61.74 \pm 2.37$ & $5.73 \pm 0.10$ & $4.41 \pm 0.08$ & $11.60 \pm 0.07$ & $19.33 \pm 1.86^{\mathrm{c}}$ & $1.18 \pm 0.09^{\mathrm{b}}$ \\
T4 & $57.55 \pm 4.26$ & $5.39 \pm 0.10$ & $4.32 \pm 0.15$ & $11.55 \pm 0.28$ & $20.50 \pm 2.17^{\mathrm{c}}$ & $1.17 \pm 0.13^{\mathrm{b}}$ \\
T5 & $61.86 \pm 1.36$ & $5.67 \pm 0.03$ & $4.38 \pm 0.04$ & $11.85 \pm 0.17$ & $29.67 \pm 2.91^{\mathrm{ab}}$ & $1.82 \pm 0.16^{\mathrm{a}}$ \\
T6 & $56.80 \pm 1.97$ & $5.47 \pm 0.07$ & $4.31 \pm 0.05$ & $11.77 \pm 0.17$ & $23.50 \pm 1.98^{\mathrm{bc}}$ & $1.32 \pm 0.09^{\mathrm{b}}$ \\
T7 & $56.78 \pm 3.69$ & $5.45 \pm 0.16$ & $4.22 \pm 0.13$ & $11.17 \pm 0.37$ & $23.33 \pm 2.59^{\mathrm{bc}}$ & $1.31 \pm 0.16^{\mathrm{b}}$ \\
T8 & $57.06 \pm 3.90$ & $5.65 \pm 0.15$ & $4.23 \pm 0.12$ & $11.57 \pm 0.17$ & $19.83 \pm 1.17^{\mathrm{c}}$ & $1.11 \pm 0.05^{\mathrm{b}}$ \\
\hline Significancia & $\mathrm{NS}$ & $\mathrm{NS}$ & $\mathrm{NS}$ & $\mathrm{NS}$ & $* *$ & $* *$ \\
DMSH & 13.07 & 0.48 & 0.42 & 0.95 & 8.90 & 0.479 \\
\hline
\end{tabular}

†Los datos son medias $(n=12) \pm$ error estándar. Letras distintas en la misma columna indican diferencia significativa según la prueba Tukey $(P \leq 0.05)$. $\mathrm{PF}=$ peso de fruto; $\mathrm{DP}=$ diámetro polar; $\mathrm{DE}=$ diámetro ecuatorial; $\mathrm{F}=$ firmeza del fruto; $\mathrm{NF}=$ número de fruto; $\mathrm{RP}=$ rendimiento por planta; NS = no significativo; ${ }^{* *}=$ altamente significativo $(P<0.01), \mathrm{DMSH}=$ diferencia mínima significativa honesta. 
tratamientos T1 (33.33), T2 (31.00) y T5 (29.67), mientras que la menor cantidad se obtuvo en el tratamiento T8 (19.83). Estudios previos por García et al. (2004) y Sarbadhikary y Mandal (2017) reportaron incrementos del 22.79 y 56.25 $\%$ en el número de frutos por planta de tomate al utilizar las cepas $B$. licheniformis y $B$. subtilis, respectivamente. Los aumentos en el número de fruto debido a las RPCV podría deberse a la capacidad de producción de fitohormonas, tales como: ácido indol-3-acético, ácido giberélico, zeatina, etileno y ácido abscísico (Singh, 2013).

Por otra parte, el rendimiento registrado en el tratamiento T1 resultó ser superior en 74.35 y $83 \%$ a los tratamientos sin inocular T4 y 78 , respectivamente. En cuanto a los tratamientos T2 y T5 resultaron estadísticamente iguales al tratamiento $\mathrm{T} 1$, sin embargo, mostraron diferencias significativas al resto de los tratamientos. Abdel-Monaim et al. (2012), reportaron incrementos significativos en el rendimiento por planta de tomate inoculadas de forma independiente con $B$. cereus y $B$. megaterium, respecto al testigo sin inocular. Resultados similares reportaron Gravel et al. (2007), con la aplicación de las cepas Pseudomonas putida B strain 1 en tomate cv. Trust F1 desarrollado en condiciones de invernadero. Lo anterior podría deberse a que el género Bacillus produce antibióticos, toxinas, sideróforos, enzimas líticas e inducen la resistencia sistémica, esto justifica el amplio uso de cepas de este género bacteriano como biofertilizantes para minimizar los efectos negativos en la productividad y calidad de los cultivos agrícolas causada por distintos fitopatógenos, así mismo reduciendo la contaminación de los suelos y mantos acuíferos por los agroquímicos (Villarreal-Delgado et al., 2018). De igual forma, el género Pseudomonas es usado como biofertilizantes debido a que ayuda en la producción de la enzima 1-aminociclopropano-1-carboxilato (ACC) deaminasa, sideróforos, ácido indo-acético (AIA) y la solubilización de fosfato que estimulan el crecimiento de las plantas y la producción de frutos (Shameer y Prasad, 2018).

Aunque no hubo diferencia estadística en la variable peso de fruto, el tratamiento T5 registro aumentos del peso de fruto en un $8.41 \%$ en comparación al T8 (Tabla 2). La capacidad de promover incrementos del peso de fruto en el genotipo estudiado se podría explicar en base a los mecanismos de las RPCV que influyen la producción de ácido-indol-acético y sideróforos, que son péptidos de bajo peso molecular y tienen alta afinidad con iones de $\mathrm{Fe}^{3+}$, los cuales se han reportado ser eficaces como agentes de control biológico (Palacio-Rodríguez et al., 2017), para mejorar el desarrollo de los cultivos e incrementar el peso de los frutos, por ende obtener un mayor peso de un mayor rendimiento (Babu et al., 2015).

En cuanto a la firmeza de fruto no se mostraron diferentes estadísticas significativas entre los tratamientos evaluados, sin embargo, el tratamiento $\mathrm{T} 2$ con la fertilización reducida e inoculado con $P$. lini, presentó un incremento de 2.42 y $2.24 \%$ en comparación a los tratamientos testigos (T4 y T8). Resultados similares fueron encontrados por Esitken et al. (2010) quienes tampoco encontraron diferencias significativas en frutos provenientes de plantas inoculadas con Pseudomonas BA-8, Bacillus OSU-142 y Bacillus M-3, no obstante, los frutos de plantas con Bacillus M-3 mostraron un incremento de $7.6 \%$ en comparación al testigo. La firmeza del tejido del mesocarpio en los frutos es un buen indicador de la calidad, la textura y vida útil, asimismo, frutos con mayor firmeza podrían ser más resistentes a la pérdida progresiva de sus cualidades sensoriales causada por microorganismos. Por lo tanto, las plantas inoculadas con RPCV podrían disminuir el deterioro del fruto, debido a que reducen la actividad en la pared celular de la enzima poligalacturonasa implicada en el ablandamiento del fruto (Mena-Violante et al., 2009).

En cuanto al diámetro polar y diámetro ecuatorial los mayores valores se presentaron en el tratamiento T3, con medias de 5.73 y $4.41 \mathrm{~cm}$, respectivamente, los cuales corresponden a frutos de tamaño mediano de acuerdo a la norma oficial NMX-FF-031-1997-SCFI (1992) (Tabla 2). Aunque no existió significancia en el diámetro polar y diámetro ecuatorial el tratamiento T3 superaron en 1.42 y $4.25 \%$ a los valores registrados en el tratamiento $\mathrm{T} 8$, respectivamente. En efecto, la inoculación con las RPCV generó ligeros incrementos en el tamaño de frutos, efectos que coinciden con lo encontrado por Esitken et al. (2006) quienes registraron un incremento del $1.06 \%$, en el tamaño de los frutos de plantas de cereza dulce inoculadas con la cepa bacteriana Bacillus OSU-142, en comparación con el testigo. El diámetro polar de fruto registrado en el T3 $(5.73 \mathrm{~cm})$ resultó ser superior al valor promedio $(5.26 \mathrm{~cm})$ reportado por Abdel-Monaim et al. (2012) al evaluar la calidad de tomate cv. Super Strain B coinoculado con Azotobacter sp. + B. cereus + B. megaterium más fertilización inorgánica a base de N:P:K. La co-inoculación de microorganismos benéficos generalmente aumenta la productividad de los cultivos y disminuyen las enfermedades de las plantas con respecto a la inoculación individual de un microorganismo benéfico. La mayoría de los efectos de los microorganismos individuales o en co-inoculación son aditivos, sin embargo, se ha reportado un efecto sinérgico en algunos casos (Avis et al., 2008). Con relación al diámetro ecuatorial fue similar al valor promedio reportado por Mena-Violante y Olalde-Portugal (2007) al evaluar la calidad de tomate cv. Rio Fuego inoculado con B. subtilis BEB-13 bs más la aplicación de la solución Long Ashton. Por lo tanto, aunque la respuesta en la calidad biofísica fue similar en algunas variables evaluadas; hubo una disminución del 25 \% en la concentración de la SN, lo cual puede permitir disminuir los costos en la producción del cultivo y minimizar los riesgos de contaminación ambiental generados por las aplicaciones excesivas de fertilizantes inorgánicos (Adesemoye et al., 2009). Las RPCV ayudan a la solubilización de fosfatos, la resistencia al estrés, estabilizan los agregados y mejoran el contenido de materia orgánica del medio de crecimiento, además, retienen más $\mathrm{N}$ orgánico y otros nutrimentos, reduciendo el suministro de fertilizantes a base de nitrógeno y fósforo, optimizando la liberación de los nutrimentos (Abdel-Monaim et al., 2012; Baset et al., 2012; Tabassum et al., 2017). 


\section{Fenoles y flavonoides totales}

El tomate es un fruto con un alto contenido de compuestos bioactivos como el folato, el ascorbato, los polifenoles, los carotenoides y otros nutrientes (Al-Harbi et al., 2017). De acuerdo con el análisis estadístico, la concentración de la SN e inoculación de RPCV afectaron positivamente el contenido de compuestos fenólicos totales y capacidad antioxidante $(P<0.01)$, no así para sólidos soluble totales y flavonoides totales $(P>0.05)$ (Tabla 3). En relación con los sólidos solubles son importantes para definir la calidad de los frutos maduros de tomate. En el presente estudio todos los tratamientos presentaron frutos de calidad de acuerdo a los valores de sólidos solubles, ya que el tomate para procesos industriales y consumo en fresco deben contar con un valor de 4.5 'Brix (Rodríguez et al., 2007). Respecto a flavonoides totales, los valores obtenidos en este experimento oscilaron entre 14.33 y $24.52 \mathrm{mg} \mathrm{EQ} / 100 \mathrm{~g}$ peso fresco, para los tratamientos T3 y T1, respectivamente. Siendo el tratamiento T1 el que presentó flavonoides totales más alto en comparación al resto de los tratamientos ( $P>0.05$, Tabla 3). La aplicación de bioinoculantes a base de RPCV propician un aumento en el contenido de fenoles y flavonoides en los frutos, debido que las RPCV afectan el metabolismo del azúcar y la concentracion de vitaminas (vitamina C y B9) (Bona et al., 2015).

El mayor valor de compuestos fenólicos totales se mostró en el tratamiento T5, con valores de $30 \mathrm{mg}$ de EAG/100 g peso fresco, superando en 34.58 y $41.04 \%$ a los contenidos determinados en los tratamientos testigos (T4 y T8), respectivamente. Este comportamiento coincide con lo reportado por Fan et al. (2017), quien indica que el contenido de compuestos fenólicos fue mayor en frutos de tomate provenientes de plantas inoculadas con la cepa $B$. licheniformis

Tabla 3. Valores promedio de fenoles totales, flavonoides y capacidad antioxidante en frutos de tomate cultivado con diferentes concentraciones de solución nutritiva y rizobacterias promotoras del crecimiento vegetal. Table 3. Average values of total phenols, total flavonoids and antioxidant capacity in tomato fruits grown with different concentrations of nutrient solution and plant growth promoting rhizobacteria.

\begin{tabular}{lcccc}
\hline Tratamiento & $\begin{array}{c}\text { Solidos } \\
\text { solubles }\end{array}$ & $\begin{array}{c}\text { Fenoles } \\
\text { totales }\end{array}$ & $\begin{array}{c}\text { Flavonoides } \\
\text { totales }\end{array}$ & $\begin{array}{c}\text { Capacidad } \\
\text { antioxidante }\end{array}$ \\
\cline { 2 - 5 } & ${ }^{\circ}$ Brix & $\begin{array}{c}\text { mg EAG/100 } \\
\text { g peso fresco }\end{array}$ & $\begin{array}{c}\text { mg EQ/100 } \\
\text { g peso } \\
\text { fresco }\end{array}$ & $\begin{array}{c}\boldsymbol{\mu M} \text { Trolox/100 } \\
\text { g peso fresco }\end{array}$ \\
\hline T1 & $4.95 \pm 0.09$ & $26.56 \pm 1.58^{\text {abt }}$ & $24.52 \pm 3.84$ & $31.29 \pm 2.23^{\mathrm{b}}$ \\
T2 & $5.02 \pm 0.06$ & $17.12 \pm 1.12^{\mathrm{d}}$ & $14.72 \pm 1.38$ & $27.63 \pm 3.34^{\mathrm{bc}}$ \\
\hline T3 & $5.04 \pm 0.15$ & $27.35 \pm 1.15^{\mathrm{a}}$ & $14.33 \pm 1.18$ & $46.19 \pm 2.62^{\mathrm{a}}$ \\
T4 & $4.83 \pm 0.25$ & $22.95 \pm 1.74^{\mathrm{bc}}$ & $18.45 \pm 1.81$ & $31.73 \pm 1.86^{\mathrm{b}}$ \\
T5 & $5.03 \pm 0.07$ & $30.00 \pm 0.79^{\mathrm{a}}$ & $22.48 \pm 1.60$ & $40.06 \pm 1.26^{\mathrm{a}}$ \\
T6 & $4.77 \pm 0.14$ & $26.15 \pm 1.98^{\mathrm{ab}}$ & $17.59 \pm 1.98$ & $41.21 \pm 1.03^{\mathrm{a}}$ \\
\hline T7 & $4.82 \pm 0.14$ & $20.28 \pm 0.87^{\mathrm{cd}}$ & $20.64 \pm 4.25$ & $28.85 \pm 1.97^{\mathrm{bc}}$ \\
T8 & $4.87 \pm 0.19$ & $21.27 \pm 1.67^{\mathrm{c}}$ & $18.22 \pm 1.81$ & $23.41 \pm 2.21^{\mathrm{c}}$ \\
\hline Significancia & $\mathrm{NS}$ & $* *$ & $\mathrm{NS}$ & $* *$ \\
\hline DMSH & 0.6896 & 4.8484 & 3.4532 & 6.5604 \\
\hline
\end{tabular}

† Los datos son medias $(\mathrm{n}=12) \pm$ error estándar. Letras distintas en la misma columna indican diferencia significativa según la prueba Tukey $(\mathrm{P} \leq$ 0.05).

$\mathrm{NS}=$ no significativo, ${ }^{* *}=$ altamente significativo $(\mathrm{P}<0.01), \mathrm{DMSH}=$ diferencia mínima significativa honesta. más el $50 \%$ de fertilización en comparación a los frutos de planta fertilizadas solo al $50 \%$. Es posible que las diferencias determinadas para los fenoles totales pudieran ser atribuidas a una mayor conductividad eléctrica en la SN al $100 \%$ (AlHarbi et al., 2017). La síntesis de los compuestos fenólico es una defensa de los cultivos vegetal contra un estrés tanto biótico como abiótico (Kim et al., 2010). El incremento de la conductividad eléctrica en el sistema radical causa estrés osmótico al disminuir la energía libre del agua, así afecta de manera negativa la absorción de agua y nutrimentos. Dicho efecto podría haber incrementado la biosíntesis de metabolitos secundarios, como los compuestos fenólicos los cuales aumentan la actividad antioxidante de los frutos (Sánchez et al., 2016). Los valores encontrados en el presente estudio son mayores a los valores de 10.30 a $18.46 \mathrm{mg}$ de EAG/100 $g$ peso fresco de tomate reportados por Gahler et al. (2003), quienes aplicaron diferentes procesamientos para obtener subproductos: jugo de tomate, tomate al horno, salsa de tomate y sopa de tomate, en los cuales determinaron cómo son afectados los compuestos bioactivos (vitamina C, polifenoles y capacidad antioxidante). Registraron que los subproductos son afectados de manera significativa en el contenido de fenoles totales, dichas diferencias pueden deberse al material genético, a las actividades agrícolas y procesamiento.

\section{Capacidad antioxidante}

Los valores de la capacidad antioxidante en los frutos de tomate fluctuaron entre 23.41 y $46.18 \mu \mathrm{M}$ ETrolox $100 \mathrm{~g}$ peso fresco. La mayor capacidad antioxidante se presentó en el tratamiento T3 con un valor de $46.19 \mu \mathrm{M}$ Trolox $100 \mathrm{~g}$ peso fresco, superando en $15.30 \%$ al resto de los tratamientos, mientras que el testigo T8 presentó la menor actividad antioxidante (Tabla 3). Estos resultados coinciden con lo reportado por Ordookhain et al. (2010) quienes indican que el uso de RPCV aumentan la actividad antioxidante de los frutos de tomate debido a que ayudar a mitigar los factores ambientales que inducen el estrés oxidativo en las plantas como la contaminación del aire con ozono y dióxido de azufre, el uso excesivo de plaguicidas, la sequía y la salinidad. En términos general, los resultados obtenidos coinciden a los reportados por otros investigadores que han reportado un incremento significativo en el contenido de compuestos fenólicos y capacidad antioxidantes de los frutos de tomate al aplicar diferentes géneros de cepas de RPCV tanto de forma individual o co-inoculadas al utilizar diferentes niveles de fertilización inorgánica (Ahmed et al., 2017). Los resultados del presente estudio y los descritos anteriormente sugieren que la aplicación de RPCV podría ser una alternativa para obtener frutos de tomate con mayores contenidos de compuestos fenólicos y una mayor actividad antioxidante lo cual es importante para la salud pública porque contribuye a la prevención de algunas enfermedades.

\section{CONCLUSIONES}

Las variables analizadas para la fenología del cultivo de tomate cv. Moctezuma demostraron ser influenciadas 
positivamente a la inoculación de RPCV. El rendimiento se incrementó en las plantas inoculadas con la cepa B. paralicheniformis y fertilizadas al $75 \%$ de la solución nutritiva, lo cual permite disminuir los riesgos de contaminación generados por la aplicación excesiva de fertilización inorgánicos. La actividad antioxidante fue mejor en frutos provenientes de plantas co-inoculadas con las cepas B. paralicheniformis y $P$. lini y fertilizadas al $75 \%$ de la solución nutritiva. Por lo tanto, la inoculación de RPCV es una alternativa sostenible de fertilización que permite reducir la fertilización; sin disminuir el rendimiento y la calidad del cultivo de tomate en condiciones de invernadero.

\section{REFERENCIAS}

Abdel-Monaim, M.F., Abdel-Gaid, M.A., El-Morsy, M. y El-Morsy, A. 2012. Efficacy of rhizobacteria and humic acid for controlling fusarium wilt disease and improvement of plant growth, quatitative and qualitative parameters in tomato. ESci Journal of Plant Pathology. 1(1): 39-48.

Abraham-Juárez, M., Espitia-Vázquez, I., Guzmán-Mendoza, R., Olalde-Portugal, V., Ruiz-Aguilar, G.M., Garcia-Hernández, J.L., Herrera-Isidron, L. y Núñez-Palanius, H.G. 2018. Development, yield, and quality of melon fruit (Cucumis melo L.) inoculated with mexican native strains of Bacillus subtilis (EHRENBERG). Agrociencia. 52(1): 91-102.

Adesemoye, A.O., Torbert, H.A. y Kloepper, J.W. 2009. Plant growth-promoting rhizobacteria allow reduced application rates of chemical fertilizers. Microbial Ecology. 58(4): 921929.

Ahmed, B., Zaidi, A., Khan, M.S., Rizvi, A., Saif, S. y Shahid, M. 2017. Perspectives of plant growth promoting rhizobacteria in growth enhancement and sustainable production of tomato. En: Microbial strategies for vegetable production. A. Zaidi y M.S. Khan (ed.), pp 125-149. Cham, Switzerland. Springer Nature.

Al-Harbi, A., Hejazi, A. y Al-Omran, A. 2017. Responses of grafted tomato (Solanum lycopersiocon L.) to abiotic stresses in Saudi Arabia. Saudi Journal of Biological Sciences. 24(6): 1274-1280.

Avis, T.J., Gravel, V., Antoun, H. y Tweddell, R.J. 2008. Multifaceted beneficial effects of rhizosphere microorganisms on plant health and productivity. Soil Biology and Biochemistry. 40(7): 1733-1740.

Ayers, R.S. y Westcot, W.D. 1994. Water quality for agriculture. En: Irrigation and Drainage Paper 29 Rev. 1. FAO (ed.), pp 174. Rome.

Babu, N.A., Jogaiah, S., Ito, S., Kestur, N.A. y Tran, L.P. 2015. Improvement of growth, fruit weight and early blight disease protection of tomato plants by rhizosphere bacteria is correlated with their beneficial traits and induced biosynthesis of antioxidant peroxidase and polyphenol oxidase. Plant Science. 231(1): 62-73.

Baset, M.M.A., Shamsuddin, Z.H., Wahab, Z. y Marziah, M. 2012. Effect of plant growth promoting rhizobacterial (PGPR) inoculation on growth and nitrogen incorporation of tissuecultured Musa plantlets under nitrogen-free hydroponics condition. Australian Journal of Crop Science 4(2): 85-90.

Bharti, N., Pandey, S.S., Barnawal, D., Patel, V.K. y Kalra, A. 2016. Plant growth promoting rhizobacteria Dietzia natronolimnaea modulates the expression of stress responsive genes providing protection of wheat from salinity stress. Scientific Reports. 6(1): 34768.

Bona E., Lingua G., y Todeschini V. 2016. Effect of bioinoculants on the quality of crops. En: Bioformulations: for sustainable agriculture. N. Arona., S. Mehnaz y R. Balestrini (ed.), pp 93124. India. Springer.

Bona, E., Todeschini, V., Cantamesa, S., Cesaro, P., Copetta, A., Lingua, G., Gamalero, E., Berta, G. y Massa, N. 2018. Combined bacterial and mycorrhizal inocula improve tomato quality at reduced fertilization. Scientia Horticulturae. 234(1): 160-165.

Brand-Williams, W., Cuvelier, M.E. y Berset, C. 1995. Use of a free radical method to evaluate antioxidant activity. LWT-Food Science and Technology. 28(1): 25-30.

Chiquito-Contreras, R.G., Murillo-Amador, B., ChiquitoContreras, C.J., Márquez-Martínez, J.C., Córdoba-Matson, M.V. y Hernández-Montiel, L.G. 2017. Effect of Pseudomonas putida and inorganic fertilizer on growth and productivity of habanero pepper (Capsicum chinense Jacq.) in greenhouse. Journal of Plant Nutrition. 40(18): 2595-2601.

Esitken, A., Pirlak, L., Turan, M. y Sahin, F. 2006. Effects of floral and foliar application of plant growth promoting rhizobacteria (PGPR) on yield, growth and nutrition of sweet cherry. Scientia Horticulturae. 110(4): 324-327.

Esitken, A., Yildiz, H.E., Ercisli, S., Figen Donmez, M., Turan, M. y Gunes, A. 2010. Effects of plant growth promoting bacteria (PGPB) on yield, growth and nutrient contents of organically grown strawberry. Scientia Horticulturae. 124(1): 62-66.

Fan, X., Zhang, S., Mo, X., Li, Y., Fu, Y. y Liu, Z. 2017. Effects of plant growth-promoting rhizobacteria and $\mathrm{N}$ source on plant growth and $\mathrm{N}$ and $\mathrm{P}$ uptake by tomato grown on calcareous soils. Pedosphere. 27(6): 1027-1036.

Food and Agriculture Organizaion of the United Nations FAOSTAT, Anuario estadístico de la FAO año 2014. [Consultado 02 Octubre 2017]. 2016. Disponible en: http:// www.fao.org/faostat/en/\#data/QC

Gahler, S., Otto, K. y Bohm, V. 2003. Alterations of vitamin C, total phenolics, and antioxidant capacity as affected by processing tomatoes to different products. Journal of Agricultural and Food Chemistry. 51(1): 7962-7968.

García, J.A.L., Probanza, A., Ramos, B., Palomino, M.R. y Gutiérrez Mañero, F.J. 2004. Effect of inoculation of Bacillus licheniformis on tomato and pepper. Agronomie. 24(4): 169-176.

Glick, B.R. 2012. Plant growth-promoting bacteria: mechanisms and applications. Scientifica (Cairo). 2012: 963401.

González, R.G., Espinosa, P.B., Cano, R.P., Moreno, R.A., Leos, E.L., Sánchez, G.H. y Sáenz, M.J. 2018. Influence of rhizobacteria in production and nutraceutical quality of tomato fruits under greenhouse conditions. Revista Mexicana de Ciencias Agrarias. 9(2): 367-379.

Gravel, V., Antoun, H. y Tweddell, R.J. 2007. Growth stimulation and fruit yield improvement of greenhouse tomato plants by inoculation with Pseudomonas putida or Trichoderma atroviride: Possible role of indole acetic acid (IAA). Soil Biology and Biochemistry. 39(8): 1968-1977.

Kim, G.D., Lee, Y.S., Cho, J.Y., Lee, Y.H., Choi, K.J., Lee, Y., Han, T.H., Lee, S.H., Park, K.H. y Moon, J.H. 2010. Comparison of the content of bioactive substances and the inhibitory effects against rat plasma oxidation of conventional and organic hot peppers (Capsicum annuum L.). Journal of Agricultural and Food Chemistry. 58(23): 12300-12306. 
Kloepper, J.W. y Schroth, M.N. 1978. Plant growth promoting rhizobacteria on radishes. En: Procceding of the 4th international conference on plant pathogenic bacteria (Vol. 2). Gilbert-Clorey (ed.), pp 879-882. France.

Mena-Violante, H.G., Cruz-Hernández, A., Paredes-López, O., Gómez-Lim, M.Á. y Olalde-Portugal, V. 2009. Cambios relacionados con textura de frutos y mejoramiento de la vida de anaquel por la inoculación de raíces de tomate con Bacillus subtilis BEB-13BS. Agrociencia. 43: 559-567.

Mena-Violante, H.G. y Olalde-Portugal, V. 2007. Alteration of tomato fruit quality by root inoculation with plant growthpromoting rhizobacteria (PGPR): Bacillus subtilis BEB-13bs. Scientia Horticulturae. 113(1): 103-106.

Norma Oficial Mexicana NMX-FF-031-1997-SCFI, productos alimenticios no industrializados para consumo humano. Hortalizas frescas. Tomate - (Lycopersicun esculentum Mill.). Especificaciones. [Consultado 10 Febrero 2018]. 1992. Disponible en: http://sagarpa.gob.mx/agronegocios/ Lists/Instrumentos\%20Tcnicos\%20Normalizacin\%20y\%20 Marcas\%20Colecti/Attachments/117/NMX_TOMATE.pdf

Olanrewaju, O.S., Glick, B.R. y Babalola, O.O. 2017. Mechanisms of action of plant growth promoting bacteria. World Journal of Microbiology and Biotechnology. 33(197): 1-16.

Ordookhain, K., Khavazi, K., Moezzi, A. y Rejali, F. 2010. Influence of PGPR and AMF on antioxidant activity, lycopene and potassium contents in tomato. African Journal of Agricultural Research. 5(10): 1108-1115.

Palacio-Rodríguez, R., Coria-Arellano, J.L., López-Bucio, J., Sánchez-Salas, J., Muro-Pérez, G., Castañeda-Gaytán, G. y Sáenz-Mata, J. 2017. Halophilic rhizobacteria from Distichlis spicata promote growth and improve salt tolerance in heterologous plant hosts. Symbiosis. 73(3): 179-189.

Ramakrishnan, K. y Selvakumar, G. 2012. Effect of biofertilizers on enhacement of growth and yield on tomato (Lycopersicum esculentum Mill). International Journal of Research in Botany. 2(4): 20-23.

Rodríguez, D.N., Cano, R.P., Favela, C.E., Figueroa, V.U., Álvarez, R.V.d.P., Palomo, G.A., Márquez, H.C. y Moreno, R.A. 2007. Vermicomposta como alternativa orgánica en la producción de tomate en invernadero. Revista Chapingo Serie Horticultura. 13(2): 185-192.

Rodríguez, D.N., Cano, R.P., Figueroa, V.U., Favela, C.E., Moreno, R.A., Márquez, H.C., Ochoa, M.E. y Preciado, R.P. 2009. Uso de abonos orgánios en la produccion de tomate en invernadero. Terra Latinoamericana. 27(4): 319-327.

Salas-Perez, L., González, F.J.A., Garcia, C.M., Sifuente-lbarra, E., Parra-Terraza, S. y Preciado, R.P. 2016. Calidad biofísica y nutracéutica de frutos de tomate producido con sustratos orgánicos. Nova Scientia. 8(17): 310-325.

Sánchez, H.D.J., Fostis, H.M., Esparza, R.J.R., Rodríguez, O.J.C., De la Cruz, L.E., Sánchez, C.E. y Preciado, R.P. 2016. Empleo de vermicompost en la producción de frutos de melón y su calidad nutracéutica. Interciencia 41(3): 213-217.
Sarbadhikary, S.B. y Mandal, N.C. 2017. Field application of two plant growth promoting rhizobacteria with potent antifungal properties. Rhizosphere. 3(1): 170-175.

Statistical Analysis System SAS (2004). What's New in SAS 9.0, 9.1, 9.1.2 and 9.1.2. SAS institute Inc. Cary N. C. USA.

Shameer, S. y Prasad, T.N.V.K.V. 2018. Plant growth promoting rhizobacteria for sustainable agricultural practices with special reference to biotic and abiotic stresses. Plant Growth Regulation. 84(3): 603-615.

Singh, J.S. 2013. Plant growt promoting rhizobateria potential microbes for sustainable agriculture. Resonance. 18(1): 275281.

Singh, V.K., Singh, A.K. y Kumar, A. 2017. Disease management of tomato through PGPB: current trends and future perspective. 3 Biotech. 7(4): 255.

Steiner, A.A. 1961. A universal method for preparing nutrient solution of a certain desired composition. Plant and Soil. 15(2): 134-154.

Tabassum, B., Khan, A., Tariq, M., Ramzan, M., Iqbal Khan, M.S., Shahid, N. y Aaliya, K. 2017. Bottlenecks in commercialisation and future prospects of PGPR. Applied Soil Ecology. 121: 102-117.

Toor, R.K. y Savage, G.P. 2005. Antioxidant activity in different fractions of tomatoes. Food Research International. 38(5): 487-494.

United States Department of Agriculture. United States Standards for Grandees of Fresh Tomatoes. [Consultado marzo 02, 2016]. 1991. Disponible en: https://www.ams. usda.gov/?dDocName=STELPRDC5050331

Vacheron, J., Desbrosses, G., Bouffaud, M.L., Touraine, B., Moenne-Loccoz, Y., Muller, D., Legendre, L., Wisniewski-Dye, F. y Prigent-Combaret, C. 2013. Plant growth-promoting rhizobacteria and root system functioning. Frontiers in Plant Science. 4(1): 1-19.

Villarreal-Delgado, M.F., Villa-Rodríguez, E.D., Cira-Chávez, L.A., Estrada-Alvarado, M.I., Parra-Cota, F.I. y De los SantosVillalobos, S. 2018. El género Bacillus como agente de control biológico y sus implicaciones en la bioseguridad agrícola. Revista Mexicana de Fitopatología. 36(1): 95-130.

Xiao, Y.G., Chun, E.H., Tao, L. y Zhu, O. 2015. Effect of Bacillus subtilis and Pseudomonas fluorescens on growth of greenhouse tomato and rhizosphere microbial community. Journal of Northeast Agricultural University. 22(3): 32-42.

Yuan, C.-L., Mou, C.-X., Wu, W.-L. y Guo, Y.-B. 2010. Effect of different fertilization treatments on indole-3-acetic acid producing bacteria in soil. Journal of Soils and Sediments. 11(2): 322-329.

Zhishen, J., Mengcheng, T. y Jianming, W. 1999. The determination of flavonoid contents in mulberrey and their scavenging effects on superoxide radicals. Food Chemistry. 64(4): 555-559. 